\title{
Case Report: Early Rehabilitation Protocol for Bullae Repair and Pleurectomy: A Case Report
}

\author{
Aishwarya Gatty ${ }^{1}$ (D), Gopala Krishna Alaparthi ${ }^{2 *}$ (D, Madhav Kamath M $^{3}$ (D), Kalyana Chakravarthy Bairapareddy ${ }^{2}$ (D), Sampath Kumar
} Amaravadi ${ }^{1,4}$ (D)

1. Department of Physiotherapy, Kasturba Medical College, Mangalore Manipal Academy of Higher Education, Mangalore, India.

2. Department of Physiotherapy, College of Health Sciences, University of Sharjah, Sharjah, UAE.

3. Department of Cardiothoracic Surgery, Kasturba Medical College, Mangalore Manipal Academy of Higher Education, Mangalore, India.

4. Department of Physiotherapy, College of Health Sciences, Gulf Medical University Ajman, United Arab Emirates.

\begin{tabular}{|c|c|}
\hline $\begin{array}{l}\text { Use your device to scan } \\
\text { and read the article online }\end{array}$ & ditation: GattyA, Alaparthi GK, Kamath MM, Bairapareddy KC, Amaravadi SK. Early Rehabilitation Protocol for Bul- \\
\hline 口ifiris & $\begin{array}{l}\text { lae Repair and Pleurectomy: A Case Report. Iranian Rehabilitation Journal. 2021; 19(2):207-214. http://dx.doi.org/10.32598/ } \\
\text { irj.19.2.1169.1 }\end{array}$ \\
\hline artas & deil" http://dx.doi.org/10.32598/irj.19.2.1169.1 \\
\hline
\end{tabular}

Article info:

Received: 05 July 2020

Accepted: 17 May 2021

Available Online: 01 June 2021

\section{ABSTRACT}

Objectives: Postoperative physiotherapy is routinely prescribed for patients after thoracic surgeries. There are no established guidelines for exercise prescription after pleural resection surgeries. The present study aimed to determine the effect of an individualized postoperative physiotherapy program on a patient who underwent pleurectomy.

Methods: A 19-year-old female patient was admitted to Kasturba Medical College, Mangalore in February 2019. She was diagnosed with right spontaneous pneumothorax; then, she underwent bullae repair and pleurectomy and was given a 7-day individualized postoperative physiotherapy. The severity of dyspnoea, chest expansion, and right shoulder range of motion was recorded on postoperative day 2 and the day of discharge; the six-minute walk distance was measured on postoperative day 4 and the day of discharge.

\section{Keywords:}

Primary spontaneous pneumothorax, Thoracic surgeries, Rehabilitation, Bullae repair, Physiotherapy

Results: Significant improvements in all the outcomes were found after the individualized post-operative physiotherapy program in the reported patient.

Discussion: The provided individualized 7-day physiotherapy program improved the chest expansion, shoulder range of motion, rating of perceived exertion scores, and functional capacity in the reported patient who underwent bullae repair and pleurectomy.

\footnotetext{
"Corresponding Author:

Gopala Krishna Alaparthi, PhD.

Address: Department of Physiotherapy, College of Health Sciences, University of Sharjah, Sharjah, UAE.

Tel: +97 (15) 04899323

E-mail: galaparthi@sharjah.ac.ae
} 


\section{Highlights}

- An individualized postoperative physiotherapy protocol improved outcomes in a 19-year-old female who underwent stapling of bullae and right parietal pleurectomy.

- Improved Perme intensive care mobility score and reduced length of Intensive Care Unit (ICU) stay were outcomes achieved in the ICU.

- Increased chest expansion, functional capacity, and right shoulder range of motion were achieved at discharge from the hospital in the reported patient.

\section{Plain Language Summary}

This report described a 19-year-old girl who experienced difficulty in breathing at her college; she was detected with a condition in which air got filled in the outer covering of her right lung due to the rupture of thin-walled sacs in the lung. She underwent surgery where the rupture in the lung was repaired and the outer covering of the lung was removed. After surgery, she was treated with an individually tailored set of exercises, including breathing and mobility exercises throughout her hospital stay. Improved chest movements, right shoulder movements, and walking capacity were achieved. She was discharged from the hospital 7 days after her surgery and was requested to continue the exercises at home. These exercises helped to achieve improved outcomes and can be tailored and tried for other patients who undergo similar surgeries.

\section{Introduction}

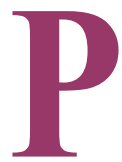

rimary Spontaneous Pneumothorax (PSP) is defined as the spontaneous occurrence of the presence of air in the pleural space in individuals without underlying lung disease. It is mostly observed in tall, thin individuals, with cigarette smoking and the male gender being the other risk factors. Loud noise, emotional changes, and atmospheric pressure alternations are the precipitating characteristics. The rupture of bulla or a subpleural bleb is believed to be the most common cause of PSP [1]. The bullae or the blebs could develop due to numerous characteristics, such as distal bronchial tree anomaly, distal airway inflammation, local ischemia, and malnutrition [2].

PSP presents with local pleuritic chest pain along with shortness of breath. Decreased breath sounds on auscultation, decreased chest wall movement on examination, and hyperresonant sound on percussion could be found during assessment [2].

Primary management is oxygen therapy or pleural drainage. Furthermore, surgical interventions are required in cases of persistent air leak or recurrent pneumothorax $[2,3]$. Surgical interventions include wedge resection, bullectomy, the stapling of bulla pleurodesis, and pleurectomy $[3,4]$. In patients undergoing thorac- ic surgeries Postoperative Pulmonary Complications (PPC) causing significant morbidity and mortality, increased hospital stay and elevated imposed costs are common. These complications could be attributed to changes in lung function, altered respiratory mechanics, reduced lung volumes, respiratory muscle dysfunction, the retention of secretions, and changes in oxygenation [5]. Physiotherapy has been found to reduce these complications [6].

Physiotherapy management for thoracic surgeries includes positioning; pain management using transcutaneous electrical nerve stimulation; early mobilization; lung expansion techniques; airway clearance techniques; inspiratory muscle training, and postural correction [7]. We presented a patient with a case of spontaneous pneumothorax who was given individualized physiotherapy protocol. This program comprised some of these techniques, post stapling of bullae, and pleurectomy.

\section{Case Report}

A 19-year-old female student, developed sudden breathlessness associated with chest pain on the right side while climbing stairs in college at the end of January 2019. She was admitted to a local hospital where the necessary investigations which included blood tests and chest X-ray were conducted. She was found to have the right pneumothorax and was managed by the insertion of 
Table 1. Physical therapy intervention protocol

Details of Interventions

- Positioning

- Lung expansion techniques

Incentive spirometer (Flow)

Diaphragmatic breathing

Thoracic expansion exercise

Ventilatory movement strategy

- Airway clearance technique splinted forced expiratory technique

- Level-wise mobilization (Figure 1)

Improving right shoulder's range of motion Active/active-assisted range of motion

Incentive Spirometer

- Post-operative Day (POD) 2,3; 5 repetitions 5 sets

- POD 4-7; 10 repetitions 3 sets

Advised to do atleast 10 repetitions every one hour

Diaphragmatic breathing

Thoracic expansion exercise

Ventilatory movement strategy

Range of motion exercises:

- Active range of motion exercises for all the limbs; 10 repetitions 1 set

- Right shoulder POD 2-4 pain-free active range of motion exercise POD 5-7

-Active Range of motion Exercise; 10 repetitions 1 set; Finger ladder exercise,

Abduction and Flexion; 5 repetitions 1 set

POD 2-7; 10 repetitions 1 set

Iranian Rehabilitation Journa

Intercostal Tube Drainage (ICD). Her breathlessness and chest pain reduced; however, the air leak persisted. Thus, after 7 days of stay in the local hospital, she was shifted to Kasturba Medical College, Mangalore in February 2019. High-Resolution Computed Tomography (HRCT) was conducted, suggesting right apical bullae. She underwent right posterolateral thoracotomy, the stapling of bullae, and parietal pleurectomy for the same.

Physiotherapy reference was given on the second postoperative day. She complained of pain at the surgical site, i.e., 8/10 on Numerical Pain Rating Scale (NPRS) and difficulty in lifting the right arm. Her heart rate was recorded as 86 beats per minute (BPM), respiratory rate: 22 BPM, peripheral oxygen saturation at rest: $100 \%$ and blood pressure were equal to $127 / 82 \mathrm{~mm} \mathrm{Hg}$. Her height was $165 \mathrm{~cm}$ and her weight was $52 \mathrm{~kg}$. No signs of respiratory distress were found at rest. On examination, chest expansion was reduced in the upper, middle, and lower zones. On auscultation, vesicular breath sounds were present but diminished all over the lung fields. Restriction in the abduction and flexion of the right shoulder was present due to pain at the surgical site. She received 2 physiotherapy sessions per day which included various interventions (Table 1).

The patient was shifted out from the Surgical Intensive Care Unit (SICU) on the third postoperative day.
Same exercises with gradual progression by increasing the number of repetitions and sets were continued. Finger ladder exercise was added to improve the right shoulder's range of motion. The drain was removed on the sixth postoperative day. The patient was discharged on the eighth postoperative day. The home program, consisting of the same interventions was taught to the patient and her company to maintain the improvements achieved during the rehabilitation.

The six-minute walk test was conducted as per the American Thoracic Society guidelines [8] on the fourth day after surgery; the test was repeated on the day of discharge. The distance was recorded on a 30-meter walk test and the vital signs were recorded as per the standard guidelines. Perme ICU mobility score [9] was used to record the mobility status of the patient in the ICU. Borg's scale for dyspnoea was applied to rate the severity of dyspnoea [10].

\section{Results}

An increase in the Perme ICU mobility score was observed post mobilization. Furthermore, the reviewed patient was shifted out of the ICU on the third postoperative day (Table 2). The intensity of breath sounds, and chest expansion also significantly improved after receiving therapy (Table 3, Figures 2, 3). The incentive spirometer values progressed from $600 \mathrm{cc} / \mathrm{min}$ without a hold on the

Table 2. The reported patient's mobility in the ICU

\begin{tabular}{ccc}
\hline Outcomes & Second Post-operative Day & Third Post-operative Day \\
\hline Perme ICU mobility score & $10 / 32$ & $27 / 32$ \\
\hline
\end{tabular}


Table 3. Chest expansion

\begin{tabular}{ccc}
\hline Zones of the Chest & Second Post-operative Day & Day of Discharge \\
\hline Upper & $1.5 \mathrm{~cm}$ & $2 \mathrm{~cm}$ \\
Middle & $1 \mathrm{~cm}$ & $2.3 \mathrm{~cm}$ \\
Lower & $1 \mathrm{~cm}$ & $3.5 \mathrm{~cm}$ \\
\hline
\end{tabular}

Пranian Rehabilitation Journal

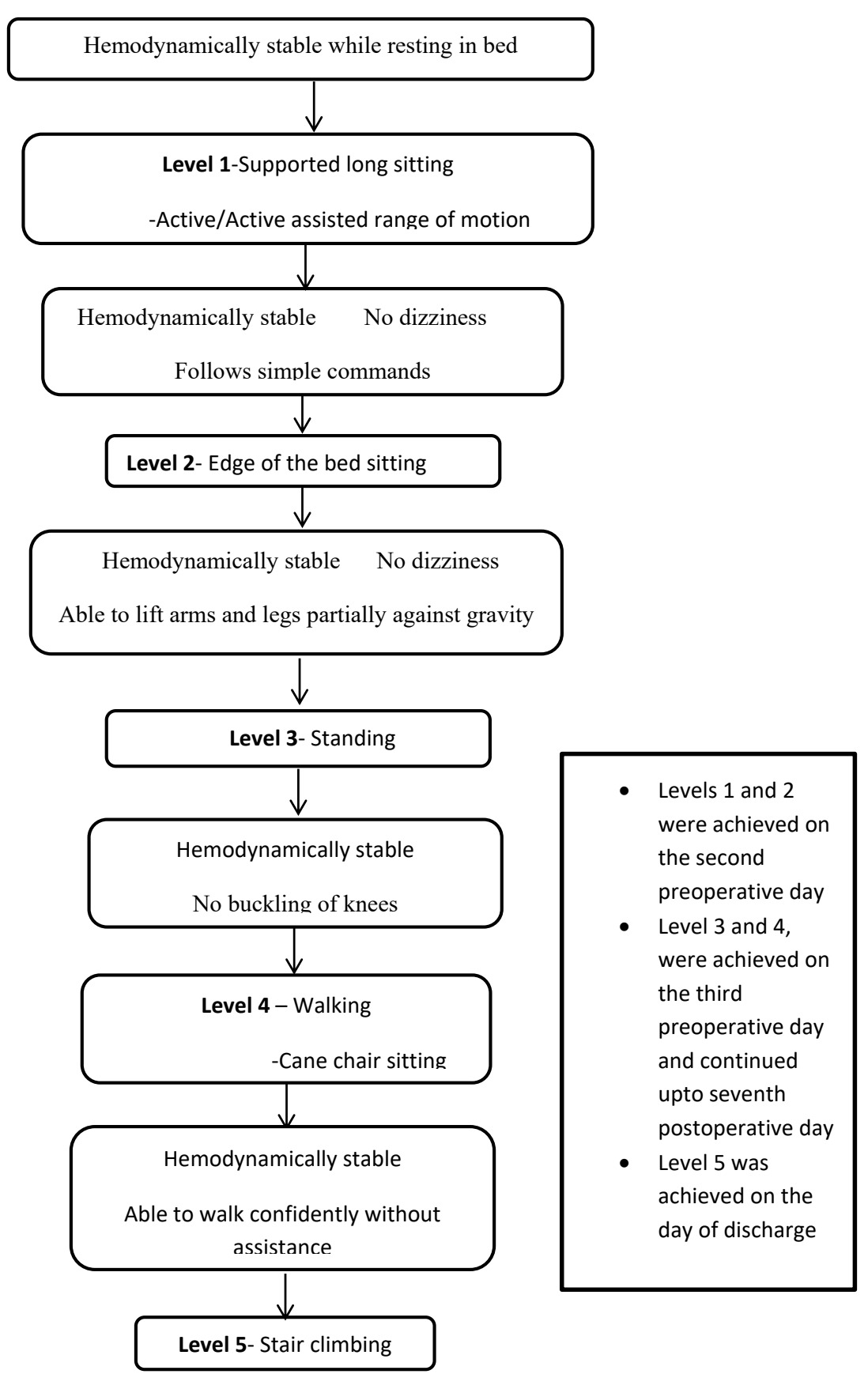

Figure 1. Level-wise mobilization

Iranian Rehabilitation Journal 
Table 4. Pain and shoulder's range of motion in the surgical side

\begin{tabular}{ccc}
\hline Parameters & Second Post-operative Day & Day of Discharge \\
\hline Pain (NPRS) & $8 / 10$ & $2 / 10$ \\
& Shoulder active range of motion & \\
Flexion & $90^{\circ}$ & $175^{\circ}$ \\
Abduction & $90^{\circ}$ & $170^{\circ}$ \\
\hline
\end{tabular}

Пranian Rehabilitation \ourna|

second postoperative day to $900 \mathrm{ccs} / \mathrm{min}$ with 2 seconds hold on the day of discharge. A decrease in the pain at the surgical site, i.e., rated $2 / 10$ on the numerical pain rating scale (NPRS) and improvement in the shoulder flexion and abduction was found after providing the therapy (Table 4). An improvement was also detected in the distance walked during the six-minute walk test, as she completed the test without any significant hemodynamic changes, minimal dyspnoea, and fatigue (Table 5).

\section{Discussion}

The reported patient underwent a 7-day postoperative physiotherapy program post-surgery. This program included lung expansion therapy, splinted forced expiratory technique, level-wise mobilization, and shoulder exercises. The patient had no post-surgical pulmonary complications; it may be attributed to the individualized physiotherapy protocol provided to the patient. Early mobilization out of the bed in the ICU improved the mobility status of the reported patient. This could have reduced the duration of ICU stay to two days. The early mobilization has been found to present positive effects in the earlier studies; the same has significantly reduced the duration of stay in ICU [11-13].

An improvement was observed in chest expansion and the right shoulder's range of motion. The improved chest expansion could be due to lung expansion exercises. These exercises, such as diaphragmatic breathing, ventilatory movement strategy, and thoracic expansion exercise are used to improve the efficiency of ventilation and gas exchange; subsequently, they increase the excursion of the diaphragm. They are also aimed to counteract an abnormal breathing pattern in the postoperative period. Thoracic expansion exercises also improve the mobility of the chest wall, trunk, and shoulder girdles. An incentive spirometer is used to prevent alveolar collapse

Table 5. Six-Minute walk test data

\begin{tabular}{|c|c|c|c|c|c|c|}
\hline \multirow{2}{*}{ Parameters } & \multicolumn{3}{|c|}{ Fourth Post-operative Day } & \multicolumn{3}{|c|}{ Day of Discharge } \\
\hline & Pretest & Posttest & $1 \mathrm{~min}$ & Pretest & Posttest & $1 \mathrm{~min}$ \\
\hline BPM (beats per minute) & 86 & 92 & 87 & 76 & 81 & 75 \\
\hline Respiratory rate (breaths per minute) & 22 & 30 & 24 & 20 & 25 & 21 \\
\hline Saturation of peripheral oxygen (\%) & 100 & 99 & 100 & 100 & 100 & 100 \\
\hline Blood pressure (mm Hg) & $122 / 80$ & $132 / 86$ & $126 / 80$ & $116 / 80$ & $124 / 84$ & $116 / 80$ \\
\hline Dyspnoea & $0 / 10$ & $4 / 10$ & $1 / 10$ & $0 / 10$ & $3 / 10$ & $0 / 10$ \\
\hline Rating of perceived exertion & $1 / 10$ & $6 / 10$ & $5 / 10$ & $0 / 10$ & $2 / 10$ & $1 / 10$ \\
\hline Laps (30 m) & & 2 & & & 6 & \\
\hline Distance $(\mathrm{m})$ & & 120 & & & 360 & \\
\hline Time (min) & \multicolumn{3}{|c|}{6 (stopped at $4 \min 45 \mathrm{sec}$ ) } & \multicolumn{3}{|c|}{6} \\
\hline Number of breaks in between the test & \multicolumn{3}{|c|}{1} & \multicolumn{3}{|c|}{0} \\
\hline
\end{tabular}




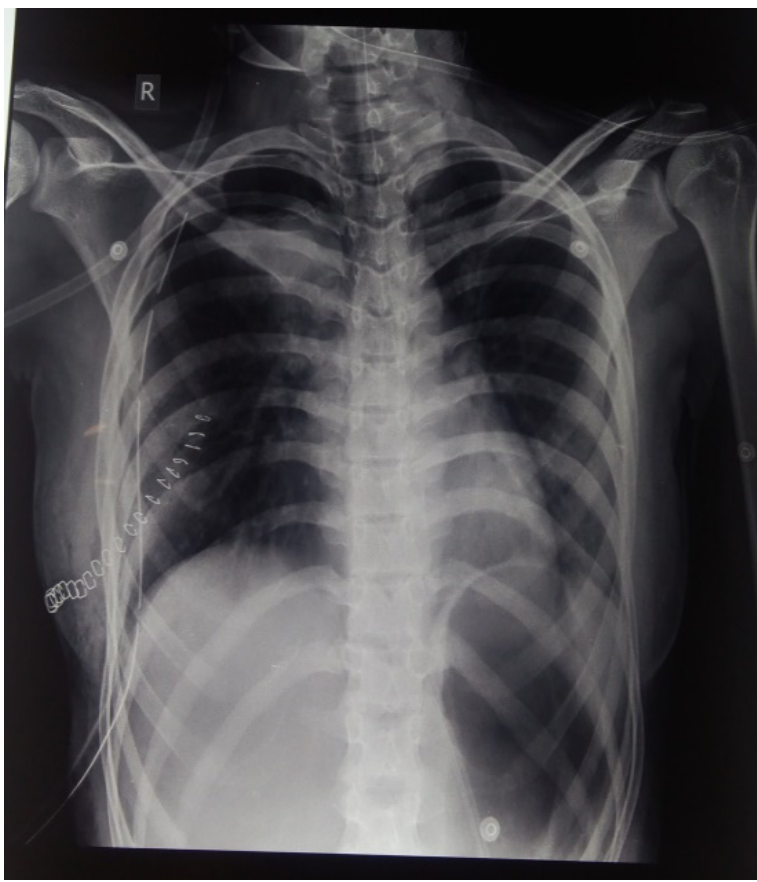

Iranian Rehabilitation Journa

Figure 2. Chest radiography on the day of surgery in the reported patient

and atelectasis in patients who have undergone surgery. Airway clearance techniques facilitate the clearance of retained secretions; thus, they prevent atelectasis and infection [14].

The improved range of motion could be associated with reduced pain due to analgesics and exercises provided for the shoulder. According to Reeve et al., a shoulder exercise program in the post-operative period improved function and decreased pain following open thoracotomy [15]. The significant changes in the distance covered during the six-minute walk test and the achievement of the last level of mobilization before discharge indicated an increase in exercise tolerance. This study signified that an individualized exercise program positively affected various outcomes of the patient after thoracotomy.

The limitations of this study were that preoperative physiotherapy and physiotherapy management for pain reduction, like transcutaneous electrical nerve stimulation was not provided to the patient. Also, analgesics and their dosage - which had and influence on the pain outcome - were not recorded. Furthermore, the long-term effect of the protocol on the patient was not assessed. Further evidence is required to determine the effect of individualized exercise programs on post-operative pulmonary complications and functional capacity in postoperative pleurectomy and other thoracic surgeries.

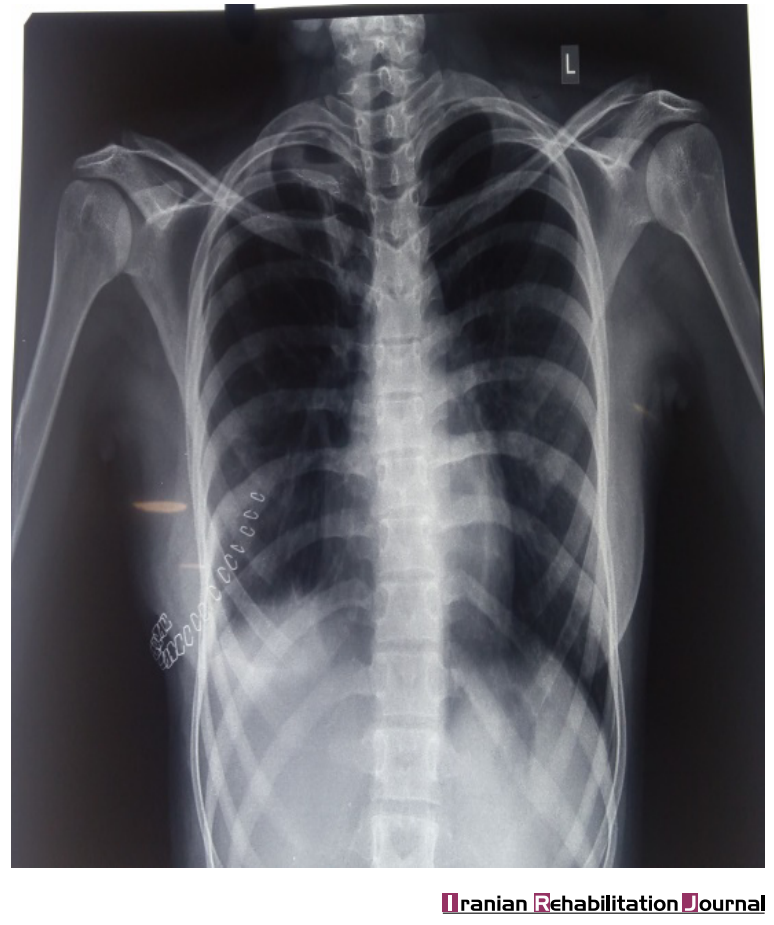

Figure 3. Chest radiography on the eighth postoperative day

\section{Conclusion}

We provided an individualized 7-day physiotherapy program that improved the six-minute walk test distance, shoulder range of motion, and chest expansion in a patient who underwent bullae repair and pleurectomy.

\section{Ethical Considerations}

\section{Compliance with ethical guidelines}

All ethical principles are considered in this article. The participant was informed of the purpose of the research and its implementation stages. She was also assured about the confidentiality of her information and was free to leave the study whenever she wished, and if desired, the research results would be available to her. A written consent has been obtained from her. The principles of the Helsinki Convention was also observed.

\section{Funding}

This research did not receive any grant from funding agencies in the public, commercial, or non-profit sectors.

\section{Authors' contributions}

All authors equally contributed to preparing this article. 


\section{Conflict of interest}

The authors declared no conflicts of interest.

\section{Acknowledgments}

The authors acknowledge the support given by the nursing staff and health professionals involved in patient care.

\section{References}

[1] Noppen M. Spontaneous pneumothorax: Epidemiology, pathophysiology and cause. European Respiratory Review. 2010; 19(117):217-9. [DOI:10.1183/09059180.00005310]

[2] Luh SP. Diagnosis and treatment of primary spontaneous pneumothorax. Journal of Zhejiang University. Science. B. 2010; 11(10):735-44. [DOI:10.1631/jzus.B1000131]

[3] Lee S, Kim HR, Cho S, Huh DM, Lee EB, Ryu KM, et al. Staple line coverage after bullectomy for primary spontaneous pneumothorax: A randomized trial. The Annals of Thoracic Surgery. 2014; 98(6):2005-11. [DOI:10.1016/j.athoracsur.2014.06.047

[4] Loubani M, Lynch V. Video assisted thoracoscopic bullectomy and acromycin pleurodesis: An effective treatment for spontaneous pneumothorax. Respiratory Medicine. 2000; 94(9):888-90. [DOI:10.1053/rmed.2000.0862]

[5] Sengupta S. Post-operative pulmonary complications after thoracotomy. Indian Journal of Anaesthesia. 2015; 59(9):61826. [DOI:10.4103/0019-5049.165852]

[6] Makhabah DN, Martino F, Ambrosino N. Peri-operative physiotherapy. Multidisciplinary Respiratory Medicine. 2013, 8:4. https:/ /link.springer.com/article/10.1186/2049-6958-8-4

[7] Ahmad AM. Essentials of physiotherapy after thoracic surgery: What physiotherapists need to know. A narrative review. The Korean Journal of Thoracic and Cardiovascular Surgery. 2018; 51(5):293-307. [DOI:10.5090/kjtcs.2018.51.5.293]

[8] ATS Board of Directors. ATS statement: Guidelines for the six-minute walk test. American Journal of Respiratory and Critical Care Medicine. 2002; 166(1):111-7. [DOI:10.1164/ ajrccm.166.1.at1102]

[9] Perme C, Nawa RK, Winkelman C, Masud F. A tool to assess mobility status in critically ill patients: The Perme Intensive Care Unit Mobility Score. Methodist DeBakey Cardiovascular Journal. 2014; 10(1):41-9. [PMID] [PMCID]

[10] Wilson RC, Jones PW. A comparison of the visual analogue scale and modified Borg scale for the measurement of dyspnoea during exercise. Clinical Science. 1989; 76(3):277-82. [DOI:10.1042/cs0760277]

[11] Adler J, Malone D. Early mobilization in the intensive care unit: A systematic review. Cardiopulmonary Physical Therapy Journal. 2012; 23(1):5-13. [DOI:10.1097/01823246201223010-00002]
[12] Zomorodi M, Topley D, McAnaw M. Developing a mobility protocol for early mobilization of patients in a surgical/trauma ICU. Critical Care Research and Practice. 2012 2012:964547. [DOI:10.1155/2012/964547]

[13] Schaller SJ, Anstey M, Blobner M, Edrich T, Grabitz SD, Gradwohl-Matis I, et al. Early, goal-directed mobilisation in the surgical intensive care unit: A randomised controlled trial. Lancet. 2016; 388(10052):1377-88. [DOI:10.1016/S01406736(16)31637-3]

[14] Frownfelter D, Dean E. Cardiovascular and Pulmonary Physical Therapy. Amesterdam: Elsevier; 2014. https:// www.google.com/books/edition/Cardiovascular_and_Pulmonary_Physical_Th/L8vsAwAAQBAJ?hl=en

[15] Reeve JC, Stiller K, Nicol K, McPherson KM, Birch P, Gordon IR, et al. A postoperative shoulder exercise program improves function and decreases pain following open thoracotomy: A randomised trial. Journal of Physiotherapy. 2010; 56(4):245-52. [DOI:10.1016/S1836-9553(10)70007-2] 
This Page Intentionally Left Blank 